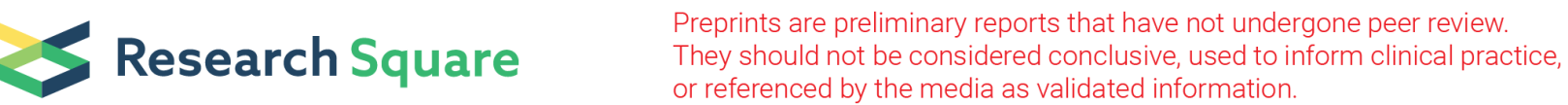

\section{One-Bone Forearm Reconstruction and Distal Radioulnar Joint Fusion for Emergency One-stage Operation in Traumatic Major Bone Defect of Forearm}

\author{
Yapeng Wang ( $\nabla$ wypsmile866@163.com ) \\ Wuxi No.9 People's Hospital affliated to Soochow University \\ Ming Zhou \\ Wuxi No.9 People's Hospital \\ Yongwei Wu \\ Wuxi No.9 People's Hospital \\ Yunhong Ma \\ Wuxi No.9 People's Hospital \\ Jun Liu \\ Wuxi No.9 People's Hospital \\ Yongjun Rui \\ Wuxi No.9 People's Hospital
}

\section{Research article}

Keywords: One-Bone forearm reconstruction, distal radioulnar joint, major bone defect

Posted Date: March 11th, 2020

DOI: https://doi.org/10.21203/rs.3.rs-16727/v1

License: (c) (1) This work is licensed under a Creative Commons Attribution 4.0 International License. Read Full License

Version of Record: A version of this preprint was published at Injury on August 1st, 2020. See the published version at https://doi.org/10.1016/j.injury.2020.06.024. 


\section{Abstract}

Background: Major bone defects in forearm caused by severe trauma is a real challenge for orthopedic surgeons. This study aimed to evaluate the role of one-bone forearm (OBF) reconstruction combined with distal radioulnar joint fusion (DRUJF) as a rescue option under this difficult situation.

Methods: In total, 18 patients with major bone defects in forearm caused by severe trauma were selected from 2003 to 2017 and followed up for 2 to 16 years. All patients were managed in the Emergency Department and received emergency one-stage OBF combined with DRUJF. In addition, patient demographics, surgical techniques, clinical outcomes and complications were collected from the medical records.

Results: The mean age of patients was 41.5 years (ranging from 23 to 58 years), with 11 males and 7 females. The mean time to union was 5.8 months; 17 patients had complete union, and 1 patient had infection with nonunion requiring secondary procedures. According to the criteria of Chen, 3 patients had a grade-I functional outcome, 14 patients had a grade-II functional outcome, and 1 patient had a grade-III functional outcome. Based on the Peterson scoring system, the outcome was excellent for 3 patients, good for 13 patients, fair for 1 patient, and poor for 1 patient.

Conclusion: OBF construction combined with DRUJF was an alternative surgical procedure as the emergency stage-one intervention for the treatment of traumatic major bone defects in forearm, which may be more functionally and cosmetically superior than forearm amputation.

\section{Background}

The bony structure of the forearm consists of the ulna, radius and associated articulations radius. The length of the ulna and radius is critical to the proper function of the forearm [1] [2]. Due to the unique rotation function of the forearm, the treatment of ulnar and radius fractures should be considered as an intra-articular fracture, which requires anatomic reduction to restore normal function. Despite advances in microsurgical technology, the treatment of major bone defects in forearm caused by severe trauma remains a real challenge. The key to treatment is the repair and reconstruction of soft tissues (including blood vessels, nerves, tendons, etc.) and bone tissues. To the best of our knowledge, there are no standard techniques described for reconstruction with major bone loss in the forearm [3]. Moreover, the loss of composite soft tissue and bone, scar adhesion, and repeated surgical procedures can lead to highly unpredictable functional outcome of this type of injury [4-6].

Under these challenging conditions, one-bone forearm (OBF) reconstruction is a feasible option that can save the limb and obtain stable and functional forearm [5-9]. The proximal ulna is generally fixed in the distal radius in OBF reconstruction [5-11]. According to the description by Vitale, "the ulna constitutes the elbow, and the radius constitutes the wrist", indicating that the ulna, as the distal extension of the arm is extremely important for elbow function, and the radius, as the proximal extension of the hand, is of great importance for the function of carpal articulation [12]. There is no consensus on the indications for this 
operation. According to previous studies, this operation is used to treat forearm bone loss caused by congenital abnormalities, tumors and infections [5-9]. However, the OBF reconstruction in the radiusconstituted wrist can generally lead to longitudinal instability in the wrist, distal radioulnar joint (DRUJ) instability and chronic pain [10]. We have also showed case reports of inferior ulnar radial dislocation after OBF reconstruction (Figure 1). Afterwards, distal radioulnar joint fusion (DRUJF) was performed as a salvage procedure. The DRUJF, similar to Sauvé-Kapandji surgery, not only enhances the stability of the wrist joint, but also attenuates the chronic pain of the wrist joints, which simultaneously has certain advantages in restoring the rotation function of the forearm [13]. Therefore, we consider whether it is possible to combine OBF reconstruction with DRUJF. To the best of our knowledge, it is the first case report with the largest sample size of the emergency treatment using OBF reconstruction combined with DRUJF to treat major bone defects in the forearm caused by severe trauma.

In this study, to our knowledge, we introduced the emergency stage-one OBF construction combined with DRUJF with the largest sample size. We aimed to assess the role of OBF reconstruction combined with DRUJF as a rescue option under this difficult situation.

\section{Methods}

A total of 18 patients were included in our retrospective analysis from 2003 to 2017 . Patients with major bone defects in the forearm caused by trauma who were willing to undergo the emergency stage-one OBF reconstruction combined with DRUJF were included in our study. Patients were excluded if they were not willing to participate in the study. In addition, patients undergoing elective procedure of OBF reconstruction and DRUJF were also excluded. The mean age of patients was 41.5 years (ranging from 23 to 58 years), with 11 males and 7 females. There were 10 cases of lesion in the right and dominant sides, and 8 cases of lesion in the left and non-dominant sides. The follow-up duration ranged from 2 to 16 years. Seven out of the 18 patients presented amputation, who required replantation; and another 11 patients had severe injuries in their limbs, who required revascularization. Seven patients were injured by work-related machine (the most common type of injury) and 4 patients had road traffic accidents. This proposal was approved by our ethic committee. The demographics of patients were shown in Table 1.

\section{Surgical technique}

All surgeries were performed by the same senior surgeon. The patient was in supine position, anesthetized with brachial plexus block, sterilized and whisked with sterile drapes in the operation area, placed with tourniquet on the upper arm, followed by debridement. Afterwards, the patient was whisked with sterile drapes in the operation area again, followed by transverse osteotomy on the ulna and radius. The length of the osteotomy is conducive to the repair of blood vessels, nerves and tendons. The hand was placed in the neutral position or pronation position not exceeding $60^{\circ}$. The fracture ends were firmly fixed with steel plates. Direct bone contact was used in all the 18 patients undergoing OBF reconstruction, which did not require iliac bone graft or free fibula transplantation. The tendon, blood vessels, and nerves were repaired accordingly. For patients who required replantation, incision decompression was 
additionally performed on opisthenar to prevent ischemia-reperfusion injury (Figure 2). Afterwards, the ulnar and radial joint was exposed, the articular cartilage cleaned was cleaned, part of cancellous bone was extracted and stuffed, 1-2 hollow nails were used to horizontally fix the ulnar and radial joint, followed by postoperative plaster fixation. Skin grafting or flap transplantation was further performed according to the situation of wound defect, active rehabilitation training was performed, and functional reconstruction was conducted if necessary.

\section{Graph review and result recording}

Patient demographics and surgical techniques were collected from medical records. After the study protocol was approved, we conducted a prospective face-to-face follow-up investigation. The following data were collected, including: limb-length discrepancy compared with the contralateral side (LLD), Chen Criteria of functional outcome [15] (Table 3), Scoring system of Peterson [6] (Table 4), Disabilities of the Arm, Shoulder and Hand (DASH) Score, grip strength: affected limb, grip strength: unaffected limb, cosmesis of forearm, time to union and complications.

\section{Results}

Seventeen of the 18 patients had complete union, and 1 patient had infection with nonunion requiring secondary procedures to achieve bone healing. The mean time to union was 5.8 months. To facilitate the primary repair of blood vessels and nerves, the ends of the bones on both sides should be shortened.

The mean forearm length of 18 patients was shortened by $7.5 \mathrm{~cm}(4.2-15 \mathrm{~cm})$ compared with the contralateral side. The upper limb functional rehabilitation training could improve the strength and function in all patients.

Functional outcome was measured by DASH scores. The mean DASH score was 12.2 points, ranging from 4.0 to 33.3 points (Table 2). According to the Chen Criteria (Table 3), the functional outcomes was divided into grade I (3 cases), grade II (14 cases), and grade III (1 case). No patient had a grade IV or required secondary amputation. The mean active wrist flexion was $43.9^{\circ}$, the mean dorsal wrist extension was $40^{\circ}$, the mean forearm pronation was $43.3^{\circ}$, and the active pronation was $42.5^{\circ}$ (Table $6)$.

The mean grip strength was $3.9 \mathrm{~kg}(1.8$ to $7.5 \mathrm{~kg})$ on the affected side and $34.2 \mathrm{~kg}$ ( 25 to $42 \mathrm{~kg})$ on the healthy side. Seventeen patients were very satisfactory with the appearance of the forearm after reconstruction, while 1 patient was somewhat satisfactory (Table 2).

Seventeen patients can resume their physical work before injury, while 1 patient failed and could only performed daily activities. One of the 18 patients had poor efficacy. Except for partial recovery of motor and sensory functions, it was mainly due to infection at the fixed site of OBF and nonunion (Table 2).

Based on the Peterson scoring system, the outcome was excellent for 3 patients, good for 13 patients, fair for 1 patient, and poor for 1 patient (Tables 4 and 5). 


\section{Discussion}

Major bone defects in the forearm caused by severe trauma is often with combined with composite soft tissue defects, and even blood supply disorders. Ischemic time is the main criterion to decide whether to preserve or amputate limbs. For severe open forearm injuries, limb preservation is superior than amputation. Daoutis et al. [16] have reported the functional outcomes of 47 patients with replantation and evaluated the outcomes using Chen criteria [15], concluding satisfactory functional outcomes in 37 of the 42 patients. In 2007, Sabapathy et al. [17] have shown the functional outcomes of 22 patients with replantation and concluded that replantation is a valuable procedure, and radical debridement, appropriate bone shortening and reduction of ischemic time are of great significance for the successful replantation. In 2017, Mattiassich et al. [18] have reported long-term results of upper limb replantation and concluded that long-term effects are possible. In this study, according to the criteria of Chen, 3 patients had a grade-I functional outcome, 14 patients had a grade-II functional outcome, and 1 patient had a grade-III functional outcome. Apart from the time of injury, segmental comminuted fractures during debridement can lead to bone loss in the radius and ulna. In consideration of the two factors, rescue is a major challenge [17] [19], and requires highly personalized therapeutic approaches.

OBF procedure has been used as a rescue option for complex forearm instability due to various causes. There is no consensus on the indications for this operation. According to previous studies, this operation is used to treat forearm bone loss caused by congenital abnormalities, tumors and infections [5-9].To our knowledge, the study by Peterson et al. [6] had the largest sample size among previous studies. In total, 19 patients received OBF reconstruction between 1973 and 1991 (only 1 case was caused by acute trauma, the rest cases were due to tumor resection / congenital abnormalities, etc.). According to the Peterson scoring system designed by Peterson et al. (the Peterson scoring system is also used in our study) (Table 4), $37 \%$ cases were excellent, $32 \%$ cases were good, $26 \%$ cases were fair and $5 \%$ cases were poor. Allende et al. [8] reported a mean clinical score of 7.7 (the Peterson scoring system [6]), without cases of infection, nonunion or re-fracture. In our case series, except for 1 patient was somewhat satisfactory, the remaining 17 patients were subjectively satisfied with limb reconstruction, and no one was willing to accept amputation, even if the limb was short and disfigured. Based on the Peterson scoring system, the outcome was excellent for 3 patients, good for 13 patients, fair for 1 patient, and poor for 1 patient. Except for 1 patient who failed to resume their original physical work and could only performed daily activities, the rest 17 patients can resume their original physical work. Therefore, OBF reconstruction combined with DRUJF is an alternative method to preserve severe acute forearm injuries and provides therapeutic ideas.

Sauvé-Kapandji procedure refers to the DRUJF and distal ulnar segment osteotomy pseudoarthrosis to treat forearm rotation dysfunction caused by changes in the distal radioulnar joint (DRUJ). SauvéKapandji procedure can relieve inferior ulnar radial arthritis or mismatch by fusing the DRUJ, resolve ulnar variability through segmental resection of the distal ulna, and restores the forearm rotation function through the formed pseudo joint [20]. Simple OBF reconstruction can generally cause wrist longitudinal instability, DRUJ instability and chronic pain [10]. Ota et al. [21] also consider that the wrist width loss 
after Sauvé-Kapandji procedure is associated with the aggravated carpal ulnar deviation, therefore, attention should be paid to maintaining the original wrist width to prevent progressive ulnar deviation of the wrist. OBF reconstruction was generally used alone. And we also found cases of distal radioulnar dislocation after simple OBF reconstruction (Figure 1). We later performed DURJF as a salvage procedure. In this study, the OBF reconstruction combined with DURJF was simultaneously performed, which can theoretically maintain the line of force in the wrist for a long time, prevent the ulnar deviation and palmar subluxation of the wrist, and decrease the number of operations.

There are certain limitations in this study. Although all data were prospectively collected, this study was a retrospective one. A prospective study involving a large population and long-term follow-up is required to confirm our results. In addition, a lack of a control group and statistical analysis in this study is correlated with the retrospective nature of this rare surgical procedure. The strengths of this study include a relatively homogeneous group of patients who suffered traumatic major bone loss in the forearm, and OBF reconstructions consist of the proximal ulna and the distal radius. Compared with other studies using telephone follow-up, face-to-face survey follow-up is used in our study, which avoids inconsistency between clinical results and patient evaluation as much as possible. Moreover, all the surgical procedures are performed by the same senior surgeon. Another advantage is the long-term follow-up, especially regarding the results of patient evaluations, which ranges from 2 to 16 years. To the best of our knowledge, it is the first case report with the largest sample size of the emergency treatment using OBF reconstruction combined with DRUJF to treat major bone defects in the forearm caused by severe trauma.

\section{Conclusion}

One-bone forearm reconstruction combined with distal radioulnar joint fusion is an alternative surgical procedure as the emergency stage-one intervention for the treatment of traumatic major bone defects in forearm, which may be more functionally and cosmetically superior than forearm amputation.

\section{Declarations}

\section{Acknowledgements}

Not applicable.

\section{Authors' contributions}

YW designed the study, prepared the manuscript. MZ and YW contributed to the data acquisition. YM contributed to the quality control of data and algorithms. JL, YR contributed to the data analysis and interpretation and reviewed the manuscript. All authors read and approved the final manuscript.

\section{Funding}


This work were supported by the Wuxi Health Management Committee (Grant No.Q201827) and the Jiangsu Natural Science Foundation (Grant No.SBK2018022454)

\section{Availability of data and materials}

The authors declare that all data supporting the findings of this study are available within the paper and its supplementary information files, or are available from the corresponding author upon reasonable request.

\section{Ethics approval and consent to participate}

The study was approved by ethics committee of Wux iNo.9 People's Hospital Affiliated to Soochow University . All patients provided signed informed consent.

\section{Conflict of interest}

The authors declare that there is no conflict of interest to disclose.

\section{References}

1. Richard M J , Ruch D S , Aldridge J M . Malunions and Nonunions of the Forearm[J]. Hand Clinics, 2007, 23(2):235-243.

2. Sarmiento A, Ebramzadeh E, Brys D , et al. Angular deformities and forearm function[J]. Journal of Orthopaedic Research, 1992, 10(1):121-133.

3. Bumbasirevic $M$, Stevanovic $M$, Lesic A , et al. Current management of the mangled upper extremity[J]. International Orthopaedics, 2012, 36(11):2189-2195.

4. Prasarn M L , Ouellette E A, Miller D R . Infected nonunions of diaphyseal fractures of the forearm[J]. Archives of Orthopaedic and Trauma Surgery, 2010, 130(7):867-873.

5. M E Castle. One-bone forearm[J]. Journal of Bone \& Joint Surgery American Volume, 1974, 56(6):1223-1227.

6. Peterson C A, Maki S, Wood M B . Clinical results of the one-bone forearm [J]. The Journal Of Hand Surgery, 1995, 20(4):609-618.

7. Lee $S \mathrm{~J}$, Jazrawi $L \mathrm{M}$, Ong B $C$, et al. Long-term follow-up of the one-bone forearm procedure[J]. American journal of orthopedics (Belle Mead, N.J.), 2001, 29(12):969-972.

8. Allende C, Allende B T. Posttraumatic one-bone forearm reconstruction. A report of seven cases.[J]. 2004, 86-A(2):364.

9. Kim S Y , Chim H , Bishop A T , et al. Complications and Outcomes of One-Bone Forearm Reconstruction[J]. Hand, 2016, 12(2):1558944716643305.

10. Jacoby S M , Bachoura A, Diprinzio E V , et al. Complications Following One-Bone Forearm Surgery for Posttraumatic Forearm and Distal Radioulnar Joint Instability[J]. The Journal of Hand Surgery, 2013, 38(5):976-982.e1. 
11. Bachoura A, Jacoby S M , Osterman A L . One-bone forearm procedure for Hajdu-Cheney syndrome: a case report[J]. HAND, 2013, 8(4):479-482.

12. Vitale $C \mathrm{C}$. Reconstructive surgery for defects in the shaft of the ulna in children[J]. Journal of Bone \& Joint Surgery-american Volume, 1952, 34 a(34 A):804-810.

13. Sanders R A, Frederick H A, Hontas R B. The Sauvé-Kapandji procedure: a salvage operation for the distal radioulnar joint.[J]. 1991, 16(6):1125-1129.

14. Chung-Wei C, Yun-Qing Q, Zhong-Jia Y. Extremity replantation. World J Surg. 1978 Jul;2(4):513-24.

15. Chen F , Culp R W , Schneider L H , et al. Revision of the ununited one-bone forearm[J]. The Journal Of Hand Surgery, 1998, 23(6):1091-1096.

16. Daoutis N K, Gerostathopoulos N, Efstathopoulos D, et al. Major amputation of the upper extremity Functional results after replantation/revascularization in 47 cases[J]. Acta Orthopaedica, 1995, 66(s264):7-8.

17. Sabapathy S R, Venkatramani $H$, Bharathi R R, et al. Technical considerations and functional outcome of 22 major replantations: (The BSSH Douglas Lamb Lecture, 2005)[J]. Journal of Hand Surgery European Volume, 2007, 32(5):488-501.

18. Mattiassich G , Rittenschober F , Dorninger $L$, et al. Long-term outcome following upper extremity replantation after major traumatic amputation[J]. BMC Musculoskeletal Disorders, 2017, 18(1):77.

19. Chuang C C , Lai J B , Cheng S L, et al. Traction Avulsion Amputation of the Major Upper Limb: A Proposed New Classification, Guidelines for Acute Management, and Strategies for Secondary Reconstruction[J]. Plastic and Reconstructive Surgery, 2001, 108(6):1624-1638.

20. Fujita S, Masada K, Takeuchi E, et al. Modified SauvÃ@-Kapandji Procedure for Disorders of the Distal Radioulnar Joint in Patients with Rheumatoid Arthritis[J]. Journal of Bone \& Joint Surgery, American Volume, 2006, 87(1):24-28.

21. Ota N, Nakamura T, Iwamoto T, et al. Radiographic parameter analysis on modified Sauvé-Kapandji procedure. J Wrist Surg, 2013, 2(1): 19-26.

\section{Tables}




\begin{tabular}{|c|c|c|c|}
\hline Table & \multicolumn{3}{|c|}{1 Demographic data of the 18 patients* } \\
\hline Case & $\begin{array}{l}\text { Age (yr } \\
\text { Sex }\end{array}$ & Injury & Bones Forming OBF \\
\hline 1 & $43, \mathrm{M}$ & Crush & proximal ulna-distal radius \\
\hline 2 & $23, \mathrm{M}$ & Accident & proximal ulna-distal radius \\
\hline 3 & $28, F$ & $\mathrm{~L}(\mathrm{ND})$ & proximal ulna-distal radius \\
\hline 4 & $45, \mathrm{M}$ & Amputation $\mathrm{R}(\mathrm{N})$ & proximal ulna-distal radius \\
\hline & $37, \mathrm{M}$ & Amputation $\mathrm{R}(\mathrm{N})$ & proximal ulna-distal radius \\
\hline 6 & $56, \mathrm{~F}$ & Amputation L(ND) & proximal ulna-distal radius \\
\hline 7 & $41, \mathrm{~F}$ & Crush & proximal ulna-distal radius \\
\hline 8 & $33, \mathrm{M}$ & Accident & proximal ulna-distal radius \\
\hline & $57, \mathrm{~F}$ & Amputation L(ND) & proximal ulna-distal radius \\
\hline 10 & $40, \mathrm{~F}$ & Amputation $\mathrm{R}(\mathrm{D})$ & proximal ulna-distal radius \\
\hline 11 & $36, \mathrm{M}$ & Crush & proximal ulna-di \\
\hline 12 & $49, \mathrm{M}$ & Accident & proximal ulna-distal radius \\
\hline 13 & $38, \mathrm{M}$ & Accident & distal radius \\
\hline & 47,F $\mathrm{F}$ & Amputation $\mathrm{R}(\mathrm{N})$ & proximal ulna-distal radius \\
\hline 15 & $44, \mathrm{M}$ & L(ND) & proximal ulna-distal radius \\
\hline 16 & $17 M$ & Crush & proximal ulna-distal radius \\
\hline 17 & $26, \mathrm{M}$ & Amputation $\mathrm{R}(\mathrm{N})$ & proximal ulna-distal radius \\
\hline & & Crush & proximal ulna-distal radius \\
\hline & & & \\
\hline
\end{tabular}

* $\mathrm{OBF}=$ one-bone forearm, $\mathrm{F}=$ Female, $\mathrm{M}=$ Male, $\mathrm{R}=$ Right, $\mathrm{L}=\mathrm{Left}, \mathrm{D}=$ dominant, $\mathrm{ND}$ =nondominant

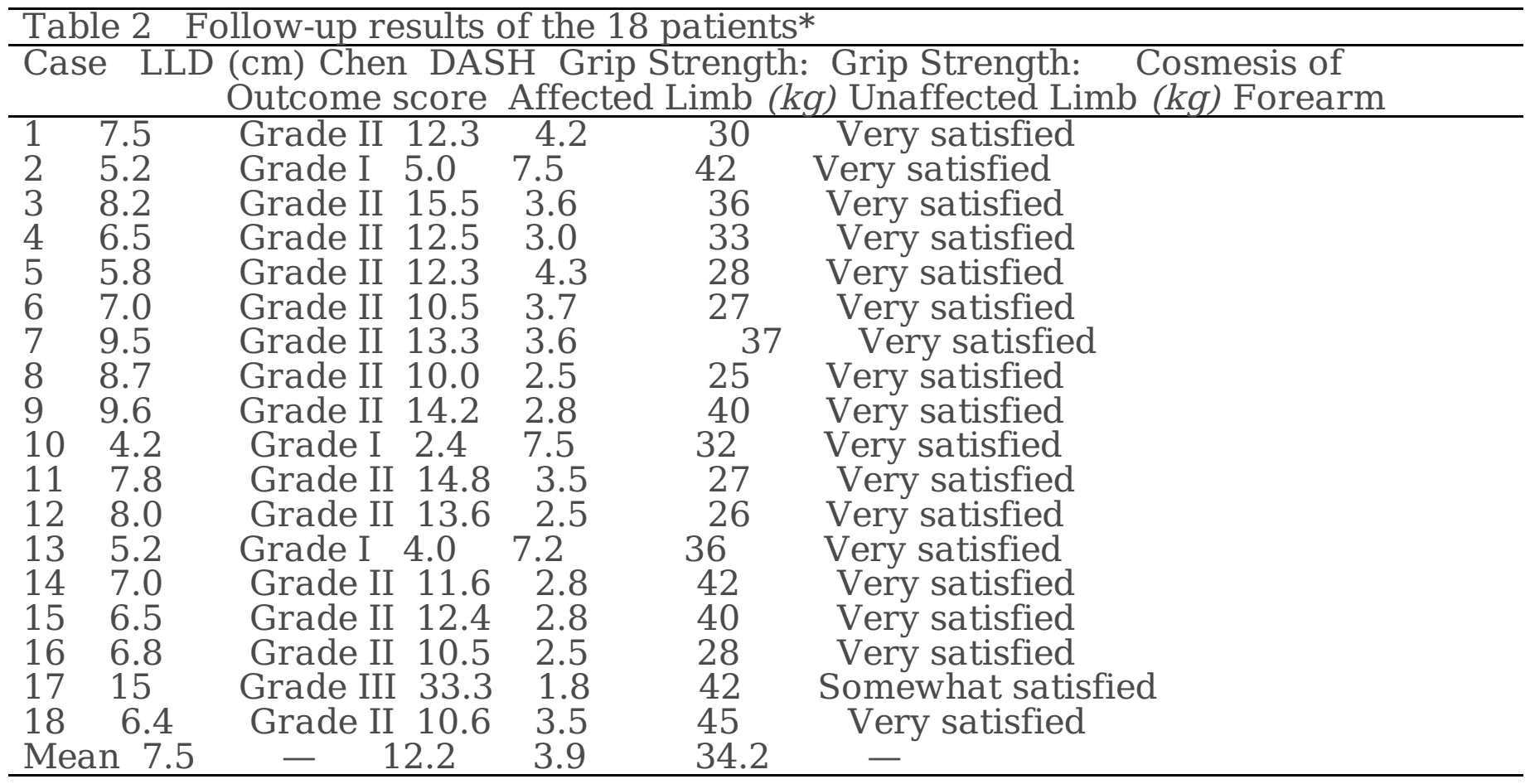

$*$ LLD $=$ limb-length discrepancy compared with the contralateral side, DASH $=$ Disabilities of the Arm, Shoulder and Hand, ADL = activities of daily living, and DRUJ = distal radioulnar joint 


\begin{tabular}{|c|c|c|c|c|}
\hline \multicolumn{5}{|c|}{ Table 2 (continued) } \\
\hline & $\begin{array}{l}\text { Return to Original } \\
\text { Occupation }\end{array}$ & Complications & 10) & Time to Union \\
\hline 1 & Yes (manual) & Nil & 5 & \\
\hline 2 & Yes (manual) & Nil & 6 & \\
\hline 3 & Yes (manual) & Nil & 4 & \\
\hline 4 & Yes (manual) & Nil & 4 & \\
\hline 5 & Yes (manual) & $\mathrm{Nil}$ & 5 & \\
\hline 6 & Yes (manual) & $\mathrm{Nil}$ & 7 & \\
\hline 7 & Yes (manual) & $\mathrm{Nil}$ & 5 & \\
\hline 8 & Yes (manual) & Nil & 6 & \\
\hline 9 & Yes (manual) & Nil & 7 & \\
\hline 10 & Yes (manual) & Nil & 6 & \\
\hline 11 & Yes (manual) & Nil & 5 & \\
\hline 12 & Yes (manual) & Nil & 5 & \\
\hline 13 & Yes (manual) & Nil & 6 & \\
\hline 14 & Yes (manual) & Nil & 4 & \\
\hline 15 & Yes (manual) & Nil & 5 & \\
\hline 16 & Yes (manual) & $\mathrm{Nil}$ & 5 & \\
\hline 17 & No, only ADL & Nonunion, infection & & 15 \\
\hline $1 \varepsilon$ & Yes (manual) & Nil & 4 & \\
\hline & - & 5.8 & & \\
\hline
\end{tabular}

Table 3 Chen Criteria of Functional Outcome in Patients with Replantation and Revascularization*

\begin{tabular}{lc}
\hline Grade & Function \\
\hline I & Able to resume original work. \\
Range of motion exceeds 60\% of normal. & Complete or nearly complete recovery of sensibility. \\
Muscle power of Grades 4 and 5
\end{tabular}

*Modified by permission from: Springer Nature. World J Surg. Extremity replantation. Chung-Wei C, Yun-Qing Q, Zhong-Jia Y. COPYRIGHT 1978. 


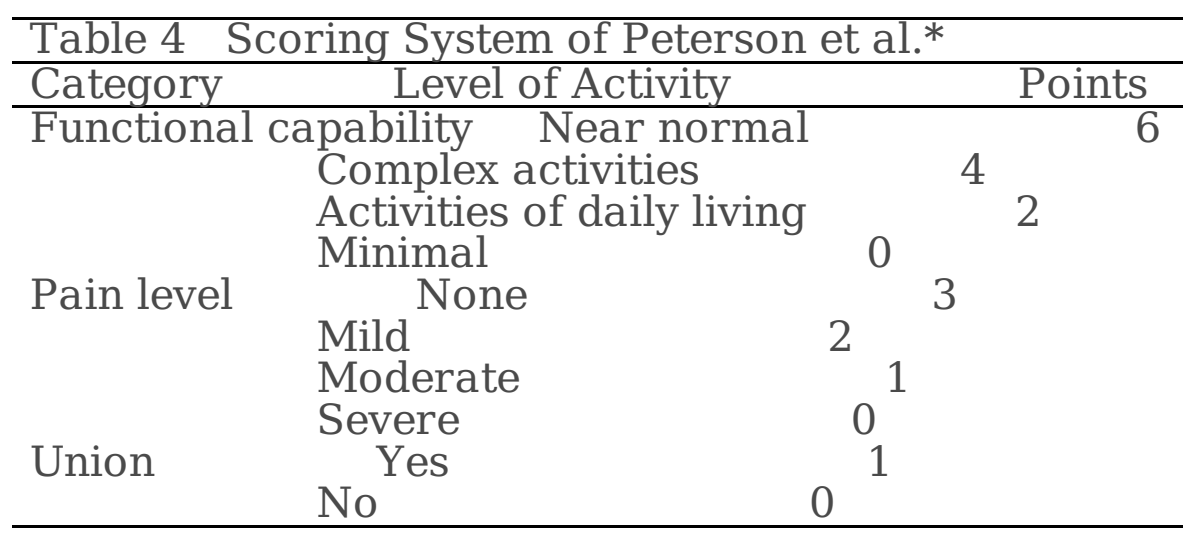

*Scoring: excellent $=8$ to 10 points, good $=6$ to 7 points, fair $=4$ to 5 points, and poor $=0$ to 3 points. (Reprinted from J Hand Surg; 20(4), Peterson CA, Maki S, Wood MB. Clinical results of the onebone forearm, 609-18, Copyright 1995, with permission from Elsevier.)

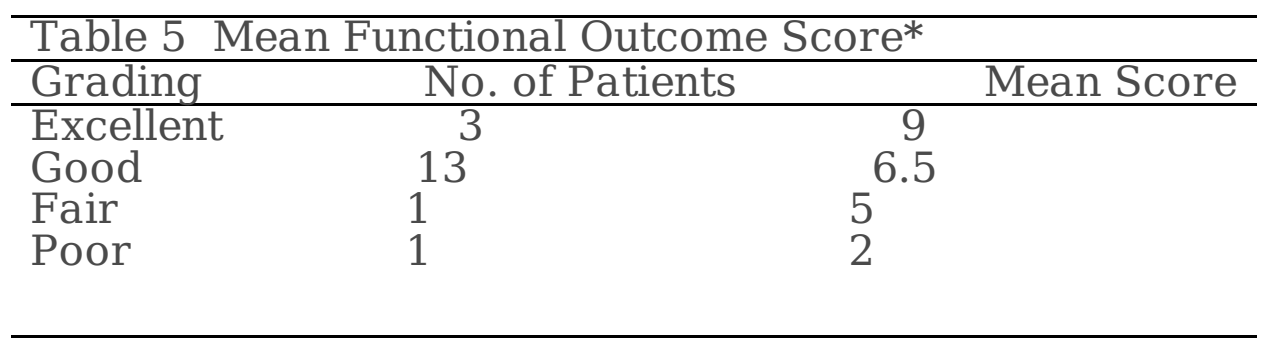

*Based on the scoring system of Peterson et al. [6]

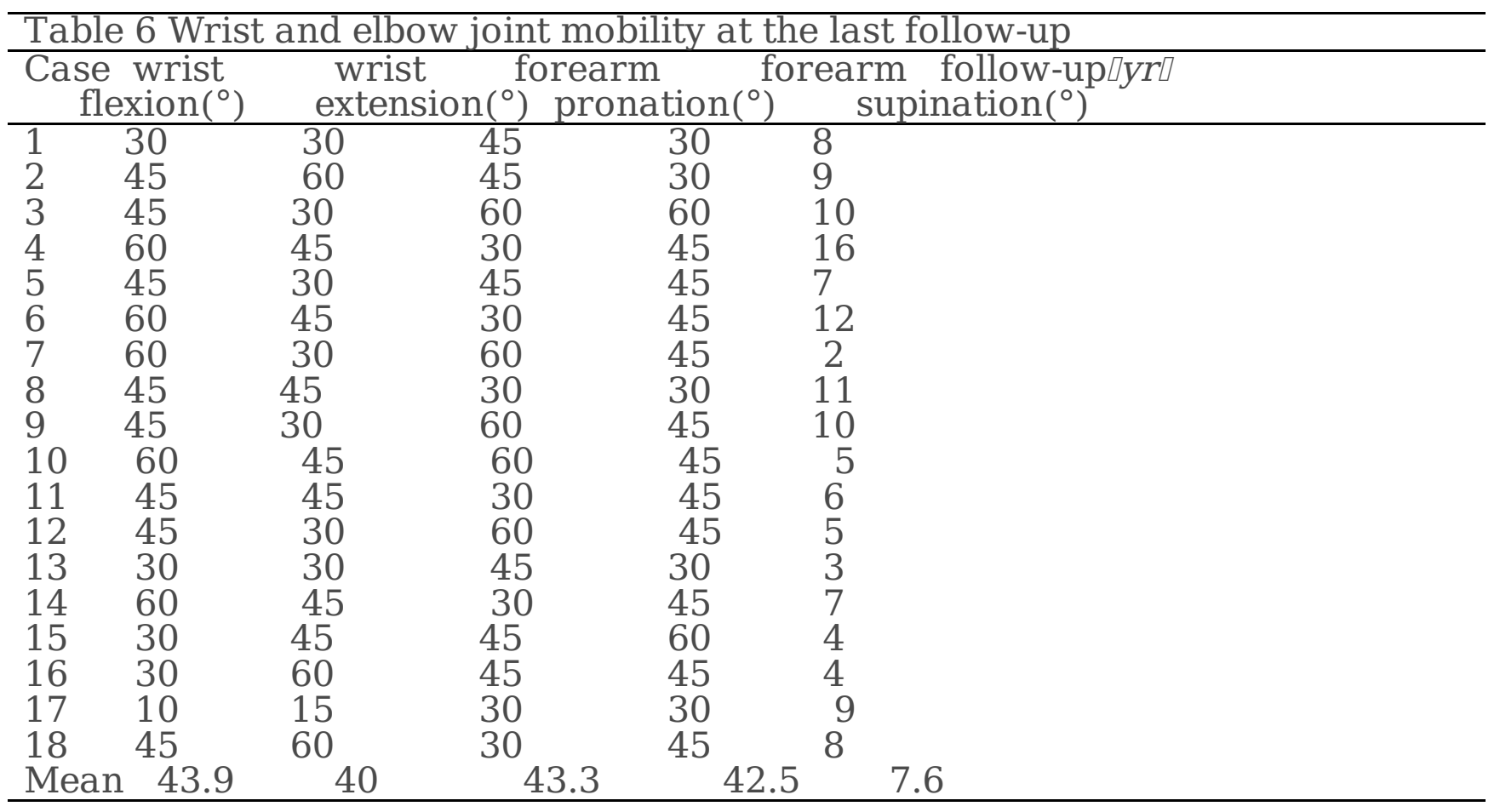


Table 7 Reports on One-Bone Forearm Reconstruction in the Literature Study Year No of Patient Results

Castle[5] $\quad 1974 \quad 6 \quad$ Good

Peterson et al.[6] $1995 \quad 19 \quad$ Poor results in 10 patients

Chen et al.[15] $1998 \quad 7 \quad$ Good

Allende and Allende[8] $2004 \quad 7 \quad$ Good

Jacoby et al.[10] $2013 \quad 10 \quad$ Average results

Kim et al [9] $\quad 2017 \quad 8 \quad$ Good

Current series $\quad 2020 \quad 18 \quad$ Good to excellent

Figures 

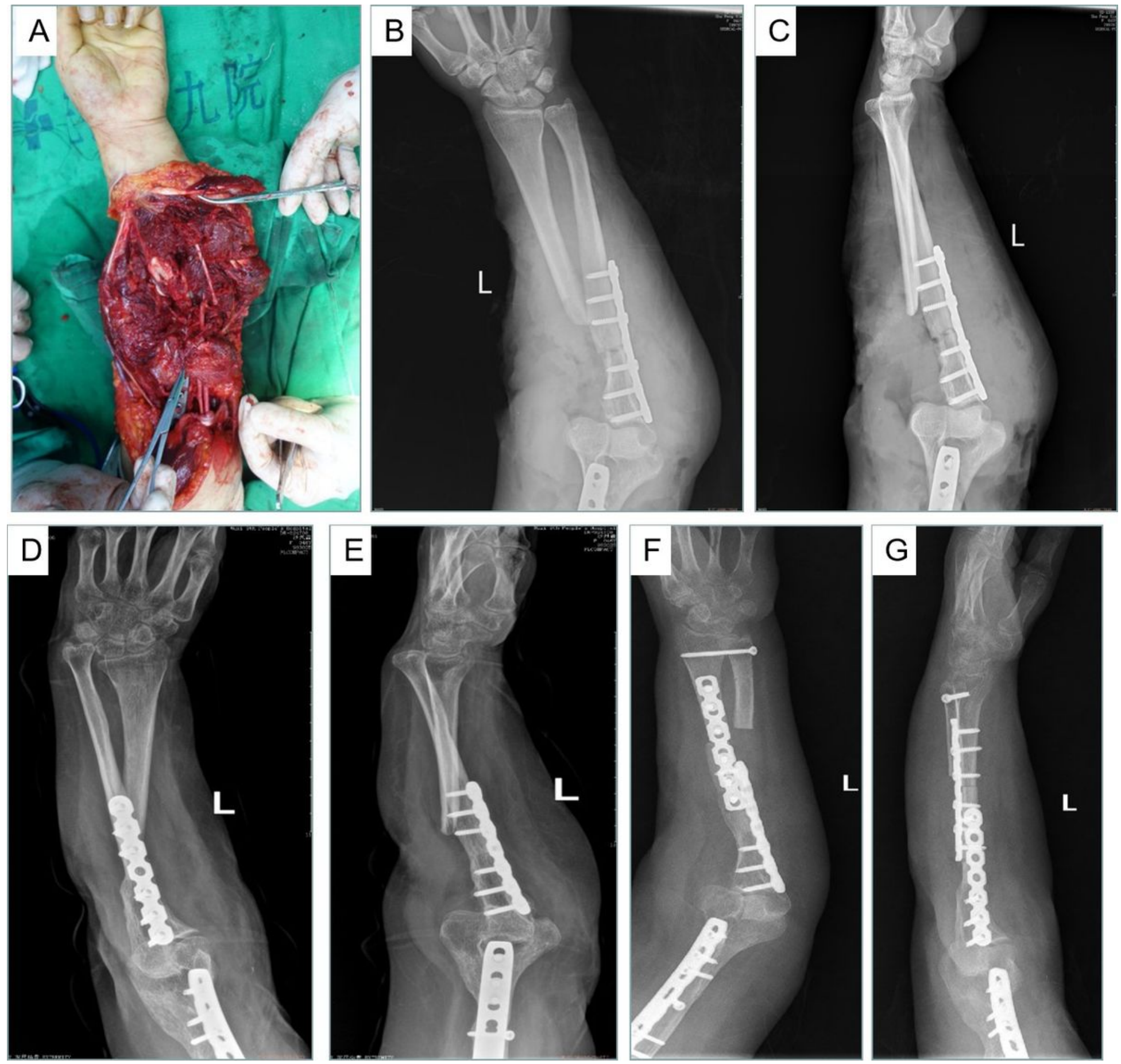

\section{Figure 1}

A 45-year-old woman with open fracture of the left ulnar radius combined with composite soft tissue defect due to mechanical crush injury received emergency stage-one OBF reconstruction. A. Appearance of injured limb; B-C. Postoperative X-ray showed OBF reconstruction of the proximal ulna and distal ulna; D-E. Distal radioulnar joint dislocation 9 months after operation; F-G. DRUJF was further performed, which was similar to Sauvé-Kapandji procedure. 

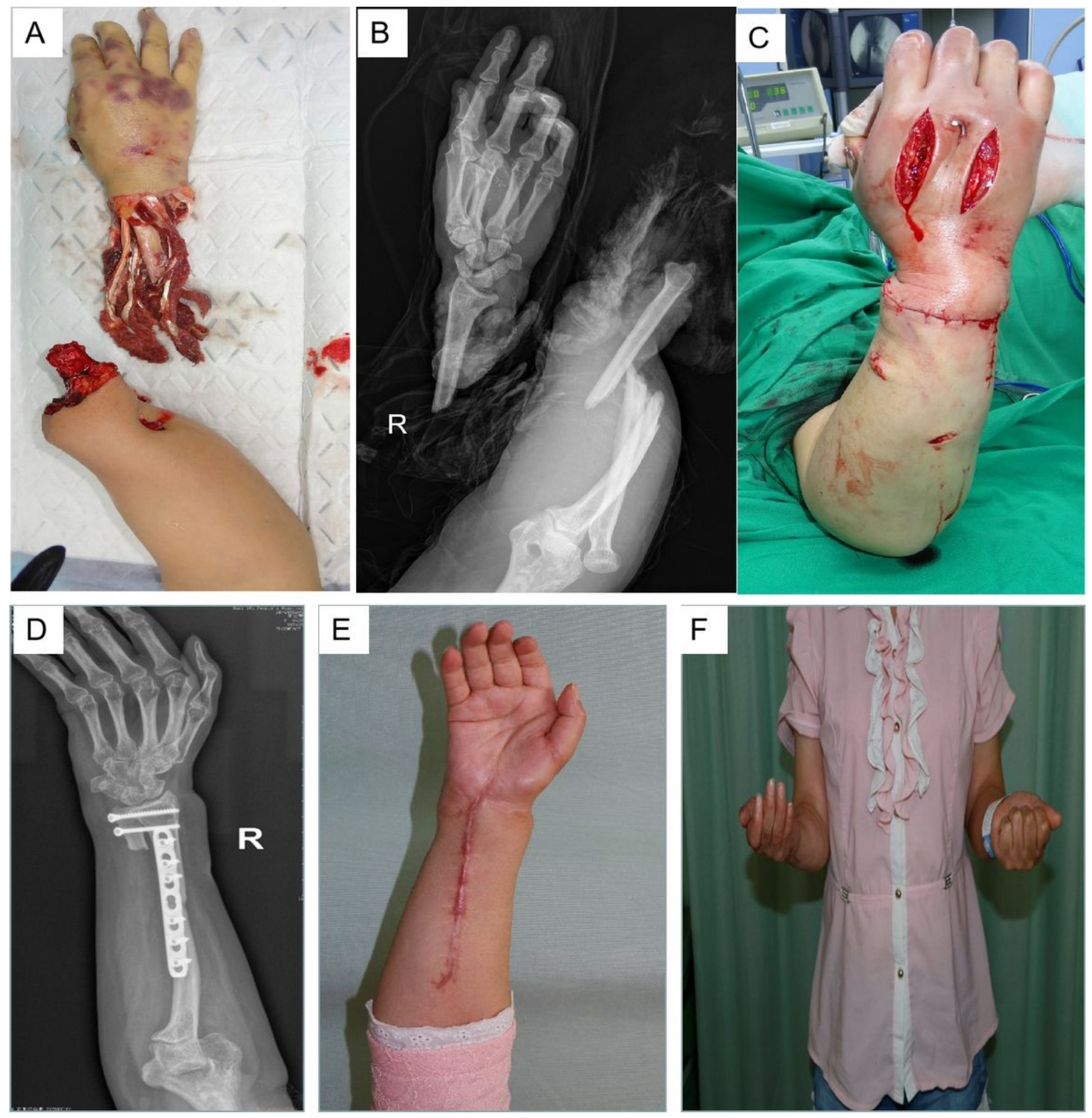

\section{Figure 2}

A 40-year-old woman suffered avulsion and detachment in the middle of her right forearm due to mechanical crush injury and subsequently received emergency stage-one OBF reconstruction combined with DRUJF. A. Appearance of injured limb; B. X line showed fracture of ulna and radius with segmental bone defect of ulna, and dislocation of radial head; $C$. Replantation of shortened forearm, end-to-end anastomosis of blood vessels and nerves, opisthenar incision decompression; D. Postoperative X-ray 
showed OBF reconstruction of the proximal ulna and distal ulna and DRUJF, which was similar to the Sauvé-Kapandji procedure; F. According to criteria of Chen, the prognosis of the patient was grade I, without complications. 